\title{
Crude oil flow modelling with the MPTT model
}

\author{
Madjid Meriem Benziane ${ }^{1, a}$, Abdelkrim Liazid ${ }^{2}$, Benyebka Bou-Said ${ }^{3}$ \\ AND Olivier Bonneau ${ }^{4}$ \\ 1 Département de génie mécanique, Université Hassiba Ben-Bouali, BP 151, Chlef, Algérie \\ 2 Laboratoire LTE ENSET-Oran, BP 1523, El Mnaouer, 31000 Oran, Algérie \\ 3 Laboratoire de Mécanique des Contacts et des Structures, INSA de Lyon, UMR 5259, 18-20 rue des Sciences, \\ 69621 Villeurbanne Cedex, France \\ 4 Laboratoire de Mécanique des Solides, Faculté des Sciences, SP2MI, Université de Poitiers, Boulevard Marie et Pierre Curie, \\ BP 30179, 86962 Futuroscope-Chasseneuil Cedex, France
}

Received 3 April 2007, Accepted 7 February 2008

\begin{abstract}
The crude oil is a non-Newtonian fluid exhibiting, during its flow, phenomena which cannot be described with the traditional models which consider the fluid as homogeneous. Thus, it is necessary to use or develop a more realistic approach which can take into account the microstructure characteristics of crude oils in the law of behaviour. The application of a non-linear differential model in the case of crude oil flow can not be used. This paper deals with the modelling approach resulting from rheology of the polymer solutions. The Modified Phan-Thien and Tanner model (MPTT) is considered in this paper for an axisymmetric flow in a pipe. The shear stresses calculation with this model is an important step as it allows to study subsequently some important phenomena as the corrosion or the pressure drop which is controlled by the friction factor under turbulent flow conditions. The calculations were about different crude oils and the obtained results are compared to experiments. Some discussions are given.
\end{abstract}

Key words: Rheology / crude oil / non-Newtonian / flow modelling

Résumé - Modélisation de l'écoulement du pétrole brut avec le modèle MPTT. Le pétrole brut est un fluide non-newtonien présentant durant son écoulement des phénomènes qui ne peuvent pas être représentés par des modèles traditionnels qui considèrent l'hypothèse d'un fluide homogène. Ainsi, il est nécessaire de prendre en compte les caractéristiques de la microstructure du pétrole brut dans la loi du comportement, approche plus réaliste. L'application du modèle différentiel non-linéaire dans le cas de l'écoulement du pétrole ne peut être utilisée. Cet article étudie une approche de modélisation résultant de la rhéologie des solutions des polymères. Le modèle de Phan-Thien et Tanner Modifié (MPTT) est considéré dans cette étude pour un écoulement axisymétrique dans un conduit. Le calcul des contraintes de cisaillement avec ce modèle est un pas important parce qu'il permet d'étudier en conséquence quelques phénomènes importants tels que la corrosion ou la chute de pression qui est contrôlée par le coefficient de frottement dans le cas d'écoulements turbulents. Les calculs concernent différents cas de pétrole brut et les résultats obtenus sont confrontés à des données expérimentales. Enfin, quelques discussions sont effectuées.

Mots clés : Rhéologie / pétrole brut / non-newtonien / modélisation d'écoulement

\section{Introduction}

The relation between the rheological properties and the crude oils structure is fundamental. The crude oil flow generates normal and tangential stresses. As their measurements are difficult to perform, the modelling technique can be used, allowing, in the most realistic possible context, the evaluation of physical properties, as shear stresses at the wall. These stresses are very important to

\footnotetext{
${ }^{a}$ Corresponding author: mbmadid20010yahoo.fr
}

estimate for corrosion study [1], friction factor determination under turbulent flows [2] and global wall-fluid interactions. The crude oil flow is very particular and cannot be represented by the traditional models. The simplest approach is to consider the crude oil as a homogeneous fluid: these are the viscosity models which consist only in taking into account the viscosity variation according to the rate of shearing. The diluted suspensions theory constitutes the first stage towards the consideration of crude oil micro-structural characteristic. The crude oil is considered as a solution of rigid particles in different solvents. 


\section{Nomenclature}

\begin{tabular}{|c|c|c|c|}
\hline Symbol & Designation & Symbol & Designation \\
\hline$D$ & Deformation rate tensor & $\lambda_{i}$ & Relaxation time of the fluid \\
\hline$g_{i}$ & $\begin{array}{l}\text { Stability sensitivity } \\
\text { coefficients }\end{array}$ & $\sigma_{i}$ & Normal stress \\
\hline$G_{i, \theta}$ & Shearing modulus of crude oil & $\rho$ & $\begin{array}{l}\text { Chain joining two successive } \\
\text { junctions }\end{array}$ \\
\hline$h_{i}$ & Chain size function & $\dot{\rho}$ & Velocity of transformation \\
\hline$k$ & Boltzman constant & $\Gamma$ & $\begin{array}{l}\text { Time dimension parameter fixed } \\
\text { numerically to } 11.08^{*} \lambda\end{array}$ \\
\hline$k_{i}$ & Formation rate of $i$-chains & $\psi_{i}(\rho)$ & $\begin{array}{l}\text { Distribution function of end-to-end } \\
\text { vectors }\end{array}$ \\
\hline$L$ & $\begin{array}{l}\text { Velocity gradient tensor of the } \\
\text { macroscopic flow }\end{array}$ & $\psi_{i, 0}$ & Function of distribution \\
\hline$l_{i}$ & Disappearance rate & $\frac{\Delta P}{L}$ & Pressure drop \\
\hline$m$ & $\begin{array}{l}\text { Exhibitor which depends on } \\
\text { the properties of the } \\
\text { suspended particles }\end{array}$ & $\tau_{\mathrm{c}}$ & Critical shear stress \\
\hline$V(r)$ & Velocity profile & $\tau_{\mathrm{w}}$ & Wall shear stress \\
\hline$N_{1}$ & First normal stress difference & $\tau$ & Shear stress \\
\hline$N_{i}$ & $\begin{array}{l}\text { Second normal stress } \\
\text { difference }\end{array}$ & $\tau^{\prime}$ & Partial stress \\
\hline$N_{i, 0}$ & $\begin{array}{l}\text { Density number of chains } \\
\text { containing } i \text { subunit }\end{array}$ & $\eta_{\mathrm{rel}}$ & $\begin{array}{l}\text { Relative viscosity given by the } \\
\text { Krieger \& Dougherty law }\end{array}$ \\
\hline$p_{i}$ & Isotropic pressure & $\eta_{\mathrm{m}}$ & $\begin{array}{l}\text { Fluid viscosity given by the } \\
\text { Krieger \& Dougherty law }\end{array}$ \\
\hline$R$ & Radius & $\eta_{0}$ & $\begin{array}{l}\text { The zero rate shearing viscosity of } \\
\text { the crude oil }\end{array}$ \\
\hline$\varsigma$ & $\begin{array}{l}\text { Velocity gradient tensor of } \\
\text { local flow }\end{array}$ & $\eta_{\infty}$ & $\begin{array}{l}\text { The limit rate shearing viscosity of } \\
\text { the crude oil }\end{array}$ \\
\hline$r$ & Radius variable & $\eta_{\mathrm{p}}$ & Viscosity of polymer \\
\hline$t$ & Flow time & {$[\eta]$} & Intrinsic viscosity $(2.5)$ \\
\hline$T$ & Temperature & $\eta_{\text {ap }}$ & Apparent viscosity \\
\hline$x_{i}$ & $\begin{array}{l}\text { Define the fraction relating to } \\
\text { the equilibrium of } i \text {-chains }\end{array}$ & $\phi$ & Effective volume fraction \\
\hline$u_{r}, u_{z}, u_{\theta}$ & Fluid velocity components & $\phi_{\mathrm{m}}$ & $\begin{array}{l}\text { Maximum volume fraction of } \\
\text { packing }(0.63-0.64)\end{array}$ \\
\hline$U_{\max }$ & Maximum velocity & $\phi^{*}$ & Dimensionless volume fraction \\
\hline$U_{\text {moy }}$ & Mean velocity & $\in$ & Constant (MPPT law) \\
\hline$\eta_{\text {apm }}$ & Modified apparent viscosity & $\xi$ & $\begin{array}{l}\text { Parameter defining the friction } \\
\text { rate }\end{array}$ \\
\hline
\end{tabular}

The non-linear differential models allow considering the flow as unstable. Lastly, a very significant branch of rheology is interested with the solutions of polymers. Indeed, there are many analogies in the flow behaviour between these polymer solutions and crude oils.

\section{Relevance of the study}

The crystallisation of wax in crude oils has been investigated for more than 30 years, first because of the severe difficulties that it causes in pipelining and storage and second because evaluation of the flow properties of oils containing wax is difficult. Initially, poor agreement was found between different rheological measurements and between laboratory and pilot plan data. During the last years, however, substantial progress has been made thanks to the efforts for the determination of the appropriate measuring techniques and to the systematic study of the physical parameters affecting the rheology. The role of the shear history on the flow properties, in particular, was clearly put into evidence. At high 


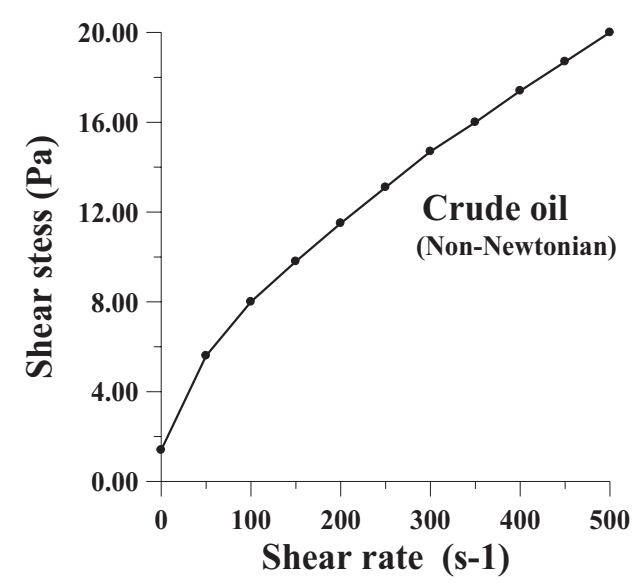

Fig. 1. Rheological behaviour of crude oil [2].

temperatures, the crude oils containing linear paraffins behave like Newtonian liquids, the paraffins being in the molten state. Below the Wax Appearance Temperature (WAT), this corresponds to incipient crystallisation of the paraffins during cooling in static conditions, oils turn into gels. The amount of solid wax responsible for gelation was recognised to be very low, around 2-4\% [2]. Cooling under flow leads to different states, which are nonNewtonian fluids, with a very rapid increase in viscosity below the WAT.

\section{Crude oil description}

Crude oil is a complex fluid in perpetual renewal, ensuring of multiple functions essential to the industrial maintenance. Different composite is an aqueous solution $(99.2-99.5 \%)$. Water occupy about $0.5-0.8 \%$ of the total volume of crude oil. Deformability is, with the capacity of stacking, a significant characteristic in the dynamics of the crude oil flow. The methodology for measuring the rheological properties is now well established. To understand the complex behaviour of waxy crude oils, a more fundamental knowledge of the relationship between microstructure and rheology is, however, necessary. In addition, the influence of the molecular composition on the mechanisms of crystallisation should help to explain the differences between crude oils from various sources. This investigation was based on the following guide lines:

- In static conditions of cooling, a precise determination of the storage and loss moduli, measured under small deformations can be undertaken. The storage modulus of the gelled oils is in general very high, (106 Pa) in the range of temperatures between $80{ }^{\circ} \mathrm{C}$ and the room temperature. One can establish experimentally the relation between the amount of crystals and the shear modulus for oils with different molecular compositions.

- The morphology of the crystals is an important aspect which is in direct relation with the rheology of crude oils and which is determined by the crystallisation process.

The rheological behaviour of the studied crude oil is shown in Figure 1.

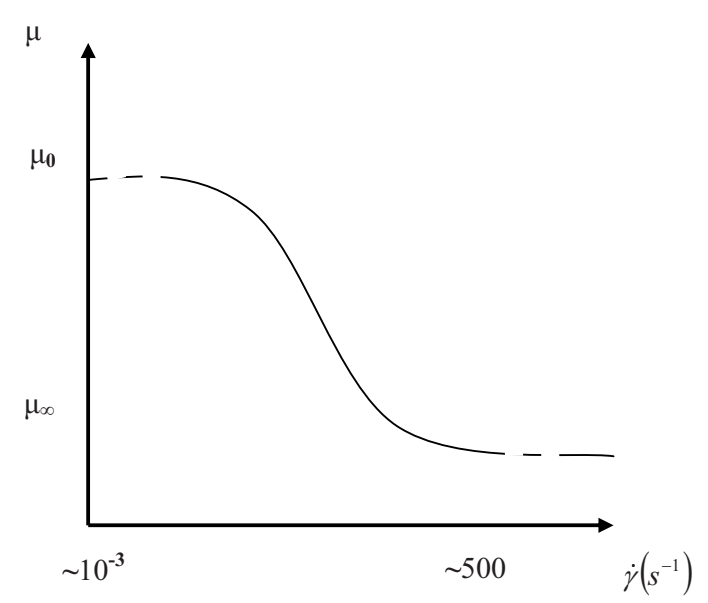

Fig. 2. Viscosity of crude oil according to the rate of shearing.

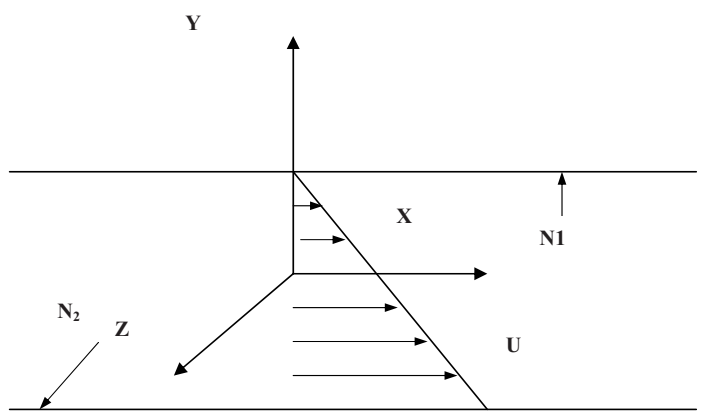

Fig. 3. Normal stresses differences.

Figure 2 shows the general shape of the viscosity evolution of crude oil [2]. This non-linearity characterizes the non-Newtonian phenomena. In addition to the characteristics of viscosity, other non-Newtonian phenomena characterize the behaviour of crude oil.

First of all, crude oil has the essential characteristic of the non-Newtonian fluids to generate normal stresses differences (or stress overshoot) during a flow in shearing. With the notations of Figure 3, the first and second stresses differences are defined by [3]: in addition, when the viscous stresses are week, the different composite agglomerate face to face by forming rollers Figure A.1, and the presence of these rollers deeply modifies the rheological behaviour of crude oil [2]. The formation of the rollers would be due to electric phenomena. The rollers assemblies are broken easily under the action of low shear stresses.

\section{Modified Phan-Thien and Tanner (MPTT) model}

The Modified Phan-Thien and Tanner (MPTT) model is today, the only model able to take into account such a broad number of specificities of crude oil and crude oil flow. This model contains nevertheless a given number of 
limitations and points which do not seem to correspond as well as possible to reality of the case of crude oil flow:

- rate of creation and rate of destruction independent of time;

- a short time of request implying negative shear stresses.

Restrictive assumption with regard to the statistical distribution: the Gaussian distribution used here is in contradiction with the fact that element are in movement.

We apply here the Modified Phan-Thien and Tanner model to the crude oil flow in a pipe. We consider the axisymmetric problem. Different crude oils are considered. Comparison with some experimental results is used for validation.

The governing equation for the Modified Phan-Thien and Tanner model (see Appendix A) is given by:

$$
\lambda_{i, 0} \frac{D \tau_{i}}{D t}+\sigma_{i}\left(\operatorname{tr} \tau_{i}\right) \tau_{i}=2 \eta_{\mathrm{m}} D
$$

with in our case [2] $\eta_{\mathrm{m}}=\eta_{\mathrm{p}}\left[1-\frac{\phi}{\phi_{\mathrm{m}}}\right]^{[\eta] \phi_{\mathrm{m}}}$ and $\sigma_{i}=$ $\exp \left(\in \frac{\operatorname{tr} \tau_{i}}{G_{i, 0}}\right)$.

\section{Flow regime}

Flow conditions in pipelines for oil production are dependent on the rheological characteristics of the hydrocarbon phase. Laminar or turbulent flow regime can be found depending on the apparent shear viscosity. Typically, liquid velocity is between 1 and $5 \mathrm{~m} . \mathrm{s}^{-1}$. At $1 \mathrm{~m} . \mathrm{s}^{-1}$, a viscosity of $100 \mathrm{cP}(0.1 \mathrm{~Pa} \cdot \mathrm{s})$ leads to a Reynolds number of the order of magnitude 1000 [2] and flow can be considered laminar. But when viscosity is around $10 \mathrm{cP}$ (0.01 Pa.s), Reynolds number is up to 10000 . So for light oil and condensate, flow regime are mainly turbulent.

\subsection{Pressure drop and shear stress at the wall}

The shear stress at the wall can be estimated from the pressure drop value. We can use a simple formulae from the force balance in steady state for pipe flow, Figure 4 which gives the following relation between stress at the wall and imposed pressure drop in the system:

$$
\tau_{\mathrm{w}}=\frac{\partial p}{\partial z} \frac{r}{2}
$$

Note that this relation is not dependent on the rheology of the system.

In the laminar regime, pressure drop calculation is related to the determination of the apparent shear viscosity of the oil. When the viscosity is known (or rheology is known if the fluid is non-Newtonian), the pressure drop calculation can be done using the equation of motion (Eq. (2)). The problem is to have the rheological knowledge of the system [2]. The stresses at the wall have a

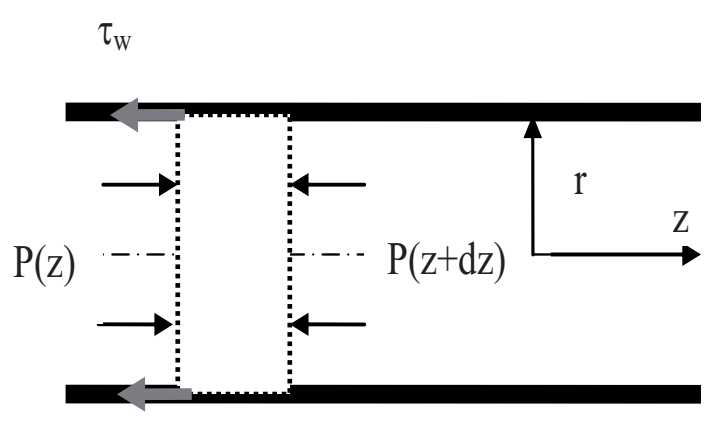

Fig. 4. Force balance on a portion of fluid.

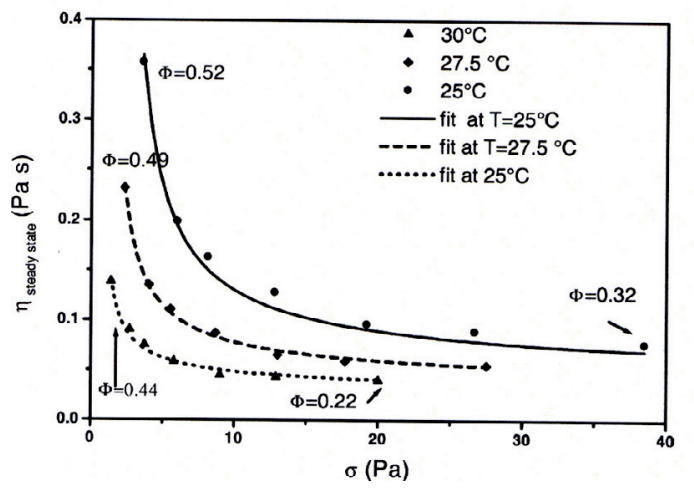

Fig. 5. The steady viscosity versus the shear stress for sheared crude oil at three temperatures. Effective volume fractions $\mathrm{F}$ at the lowest and highest shear stresses are indicated on the graphs (from [4]).

different form when flow regime is laminar or turbulent. So, the first step consists on getting the apparent viscosity law. The pressure drop $(\Delta P / L)$ in a pipe is given by [2]:

$$
\frac{\Delta p}{L}=f \frac{\rho U^{2}}{R}
$$

$U$ is the average velocity of the flow, $R$ is the pipe radius, $\rho$ the flowing phase volume mass and $f$ is only dependent upon the Reynolds number of the flow. This friction factor in the laminar Poiseuille flow case is given by: $f=\frac{16}{R_{\mathrm{e}}}$ and $R_{\mathrm{e}}=\frac{\rho U D}{\mu}$

Figure 5 gives the evolution of the steady viscosity against the shear stress for given temperatures and concentrations from [4]. After some tests using rheological data of the studied crude oils, Krieger \& Dougerty law defined by the Equation (4) seems to be the more adapted. Figures 6 and 7 show an example of results.

$$
\eta_{a p}=\eta_{\infty}+\frac{\eta_{0}-\eta_{\infty}}{1+\left[\frac{\tau}{\tau_{c}}\right]^{m}}
$$

The second step is to determine the fluid velocity profile. Thus, from the Navier-Stokes equations and apparent viscosity law from Krieger, given by Equation (4), some 


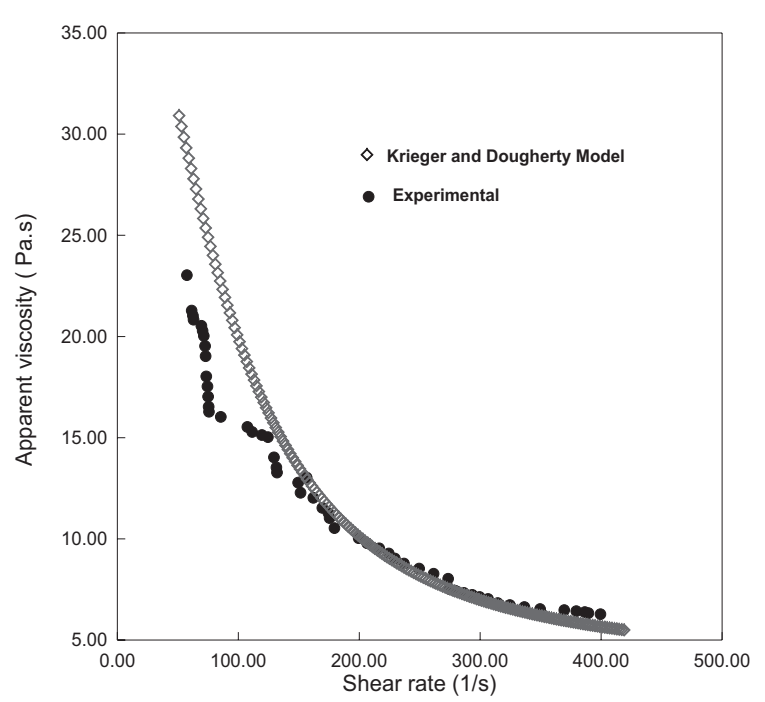

Fig. 6. Comparison between measured and calculated apparent viscosity of Asphaltenic crude oil $(\phi=0.274)$ [2].

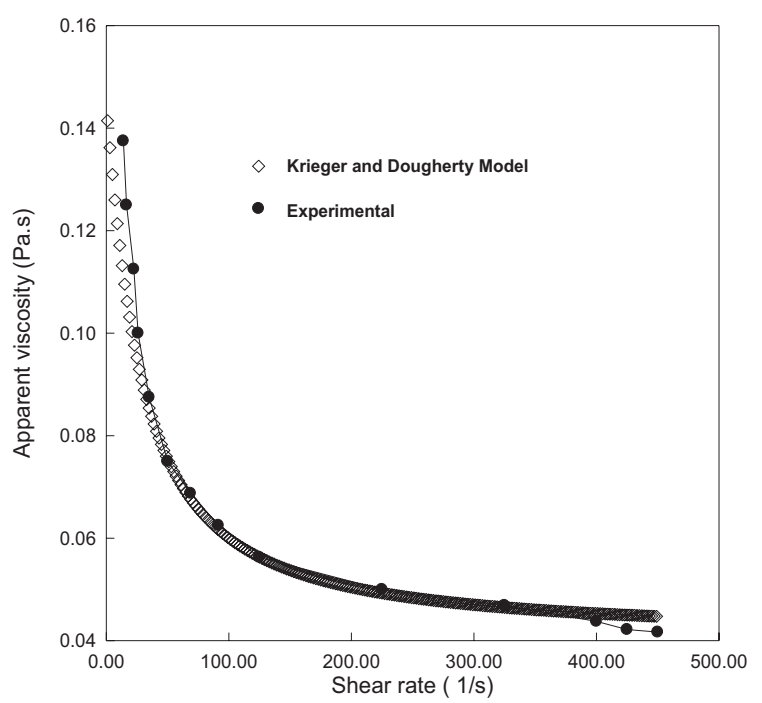

Fig. 7. Comparison between measured and calculated apparent viscosity of North Sea crude oil $(\phi=0.6)$ [4].

mathematical developments lead to the following velocity profile (Appendix B):

$$
\begin{aligned}
V(r)= & \frac{a / b^{2 / 3}}{4 \eta_{\infty}}\left(R^{2}-r^{2}\right)+\left[\frac{\left(a / b^{2 / 3}\right) A}{2 \eta_{\infty}}-\frac{\left|\tau_{\mathrm{c}}\right|}{\eta_{\infty}}\right](r-R) \\
& +\left[\frac{\left|\tau_{\mathrm{c}}\right| A}{\eta_{\infty}}-\frac{\left(a / b^{2 / 3}\right) A^{2}}{2 \eta_{\infty}}\right] \ln \left|\left[\frac{r+A}{A+R}\right]\right|
\end{aligned}
$$

with $A=\frac{2 \eta_{0}\left|\tau_{c}\right|}{a_{\infty}^{\eta}}$ and a is the pressure drop. Thus this velocity profile can be obtained for a given pressure drop.

From the velocity profile, we can compute the gradient rate tensor of the global flow $L$, the gradient rate

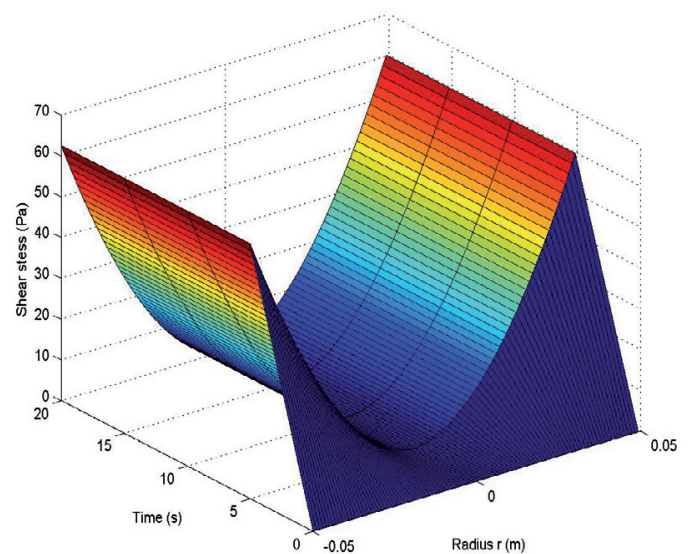

Fig. 8. Shear stress $(\tau r z)$ according to time and pipe radius. Crude oil of Venezuela [5].

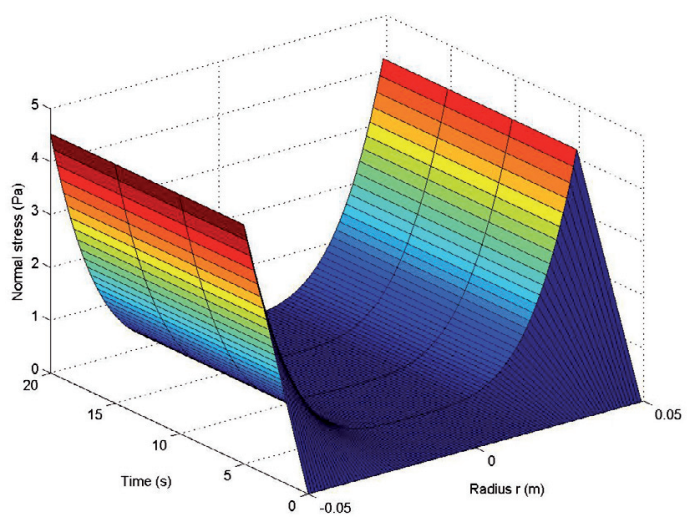

Fig. 9. Normal stress $\left(\sigma_{1}\right)$ according to time and pipe radius. Crude oil of Venezuela [5].

tensor of the local flow and the macroscopic deformation rate tensor $D$.

$$
\begin{array}{r}
L_{i j}=\frac{\partial u_{i}}{\partial u_{j}}, \quad D_{i j}=\frac{1}{2}\left(\frac{\partial u_{i}}{\partial u_{j}}+\frac{\partial u_{j}}{\partial u_{i}}\right), \quad \varsigma=(L-\xi D), \\
\frac{\mathrm{D}}{\mathrm{D} t}=\frac{\mathrm{d}}{\mathrm{d} t}()-\varsigma .()-() \cdot \varsigma^{t}
\end{array}
$$

These tensors are introduced in the constitutive equation of the studied models to obtain a system of differential equations. This system can be solved numerically.

$$
\left\{\begin{aligned}
\frac{\partial \tau_{r r}}{\partial t} & =-\frac{\sigma_{i}}{\lambda_{i, 0}} \tau_{r r}-A A \zeta \cdot \tau_{r z} \\
\frac{\partial \tau_{r z}}{\partial t} & =-\frac{\sigma_{i}}{\lambda_{i, 0}} \tau_{r z}-A A\left[\xi \cdot \tau_{z z}+(\xi-2) \cdot \tau_{r r}\right]+2 \frac{\eta_{\mathrm{m}}}{\lambda_{i, 0}} A A \\
\frac{\partial \tau_{z z}}{\partial t} & =-\frac{\sigma_{i}}{\lambda_{i, 0}} \cdot \tau_{z z}-A A(\xi-2) \tau_{r z}
\end{aligned}\right.
$$

with $A A=\frac{-\frac{a r}{2}\left[\left(\frac{a r}{2}\right)+\left(\tau_{c}\right)\right]}{\left[\left(\tau_{c}\right) \eta_{0}+\eta_{\infty}\left(\frac{a r}{2}\right)\right]}$

The system (6) is solved numerically using finite differences method.

Figures 8 and 9 represent a sample of results concerning the variation of the dimensionless shear stress and the first normal stress difference according to time and to the pipe radius, obtained with the Modified Phan-Thien 


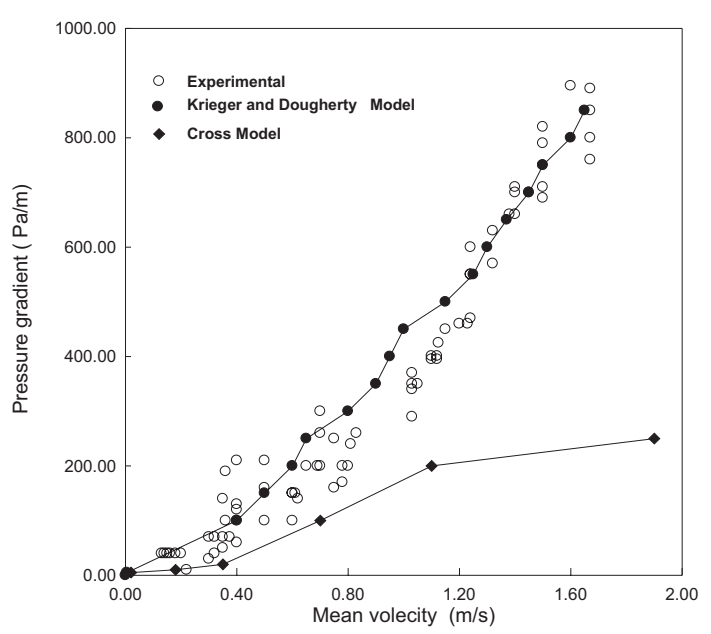

Fig. 10. Comparison between measured and calculated pressure drop according to mean velocity for naphtha and hydrate particles at $+10 \%$ water cut $\left(4{ }^{\circ} \mathrm{C}, 40\right.$ bar $)[2]$.

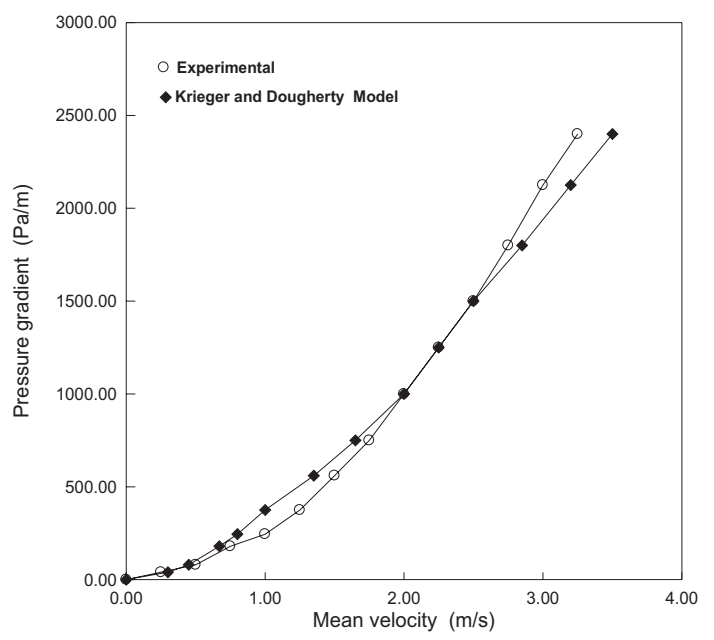

Fig. 11. Comparison between experimental and calculated pressure drop according to mean velocity for condensate and hydrate particles at $20 \%$ water cut $\left(4^{\circ} \mathrm{C}, 75\right.$ bar $)[2]$.

and Tanner model. The normal stress magnitude represents around $10 \%$ of the shear stress value. This can be a non-negligible contribution in some cases especially in transient regime where these values have the same magnitude $[6]$.

With the Phan-Thien and Tanner model we can take into account many rheological specificities of crude oils: interactions between oil and water, non-Newtonian effect,... We can also highlight the importance of the shear thinning effect. Figures 10 and 11 show an example of the experimental and calculated variation of the pressure drop according to the mean velocity for the naphtha and condensate fluids [2]. On these figures, it can be noticed that the modified Krieger \& Dougerty model is very well adapted compared to the Cross model. One can use the Krieger \& Dougerty model to get a good estimation of the pressure drop in a pipe in place of other more rough models such as Cross's model. An important information

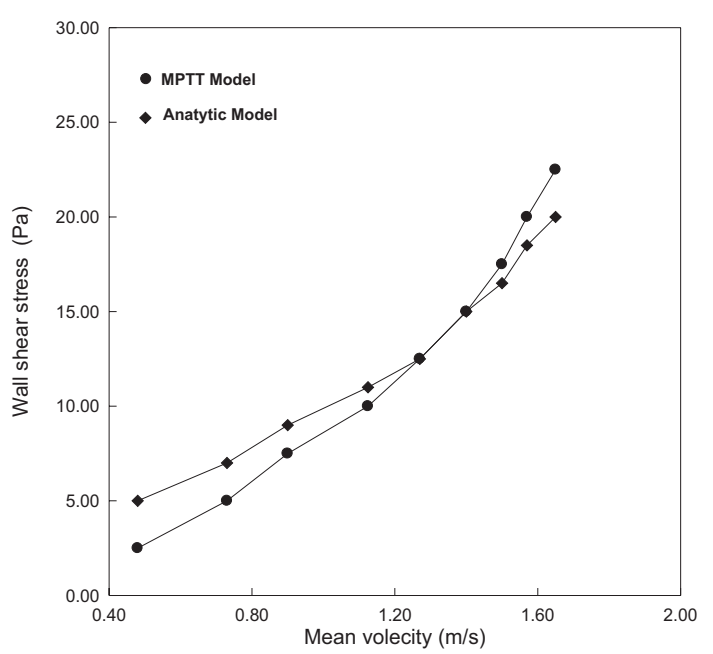

Fig. 12. Wall shear stress comparison between analytical and MPTT models for naphtha and hydrate particles at $+10 \%$ water cut $\left(4{ }^{\circ} \mathrm{C}, 40\right.$ bar $)[2]$.

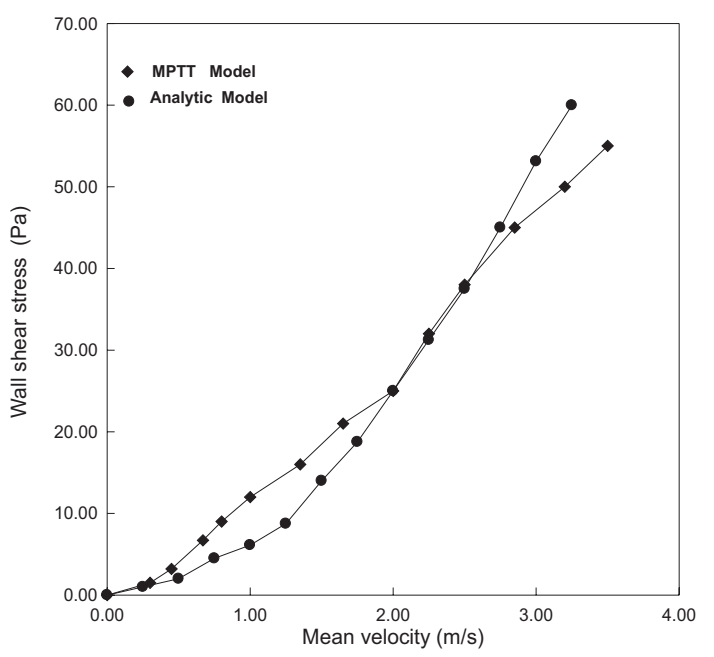

Fig. 13. Wall shear stress comparison between analytical and MPTT models for condensate and hydrate particles at $20 \%$ water cut $\left(4{ }^{\circ} \mathrm{C}, 75\right.$ bar $)[2]$.

linked to corrosion effects is the determination of the wall stress. In Figures 12 to 15, the wall shear stress results obtained with this model compared to those obtained using Equation (2) (analytical model) are similar for different crude oils. One can conclude the good concordance between these two sets of results. The Modified Phan-Thien and Tanner model predicts correctly the tendency of wall stresses. Using Equation (3) gives a correct estimation of the wall stress when compared to the values obtained from the Modified Phan-Thien and Tanner model. The prediction is better beyond the flow velocity of $1 \mathrm{~m} . \mathrm{s}^{-1}$ and notably for the Venezuelan oil. Globally the relative margin doesn't exceed $20 \%$ beyond this velocity. Using this model leads to good results with the advantages of a more physical description of the rheology of the crude oils 


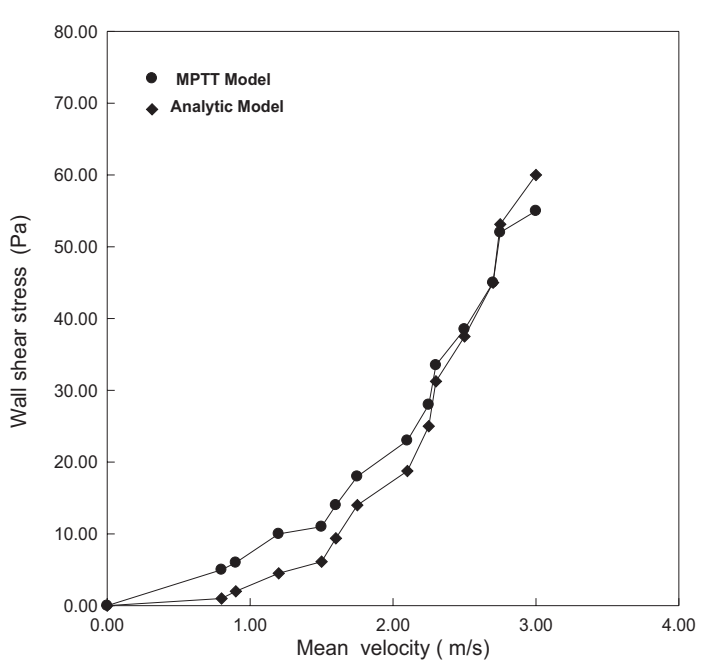

Fig. 14. Wall shear stress comparison between analytical and MPTT models for crude oil of North Sea [4].

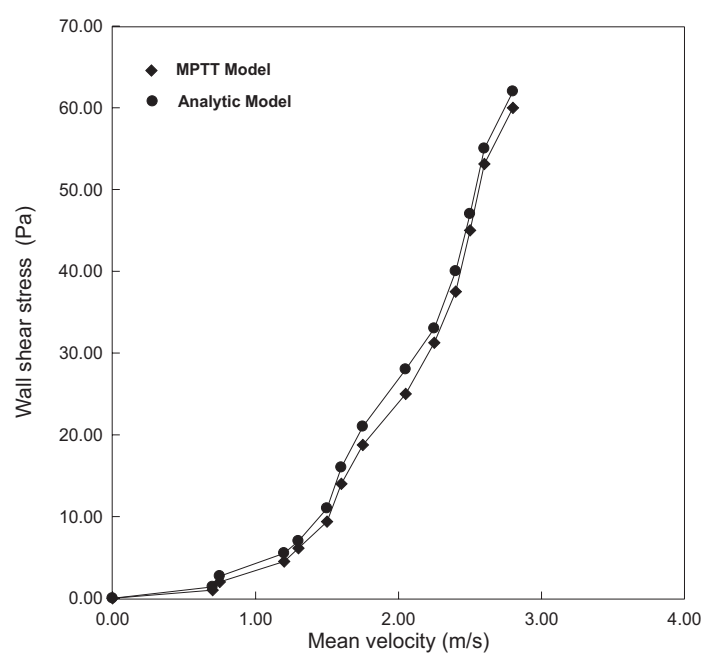

Fig. 15. Wall shear stress comparison between analytical and MPTT models for crude oil of Venezuela [5].

linking the microstructure behaviour to the macrostructure description.

\section{Conclusion}

Crude oil is a fluid presenting a non-Newtonian behavior. Its modelling is thus not easy. We decided here to use the model of Phan-Thien and Tanner, which seems in our opinion more realistic, because based on a microstructural description of the fluid. However, some assumptions of the Phan-Thien and Tanner model seem to be not representative of the phenomena observed. We observed an influence of the shear thinning effect on the stress values. It should be noticed that the model of Phan-Thien and Tanner offers the greatest flexibility on the level of the choice of configurations and boundary conditions. Next step is to apply more complex boundary conditions in order to have a better modelling of the crude oil flow.

\section{Appendix A: Phan-Thien and Tanner model}

The modern lubricants incorporate a broad variety of additives; the purpose is to improve the properties of basic oils of mineral origin. The addition of these polymers leads to a modification of the rheological behaviour of the lubricant: a linear relation between the rates of deformations and the stresses is not sufficient to describe the lubricant. Its behaviour is non-Newtonian what is mainly illustrated by the shear thinning effect. The additives imply viscoelastic effect. First of all let us point out the various choices of viscoelastic laws to describe the behaviour of the lubricant. The behaviour of the lubricant can be approached in two ways:

- By considering the lubricant as continuous, viscoelastic medium: Theory of the continuous mediums;

- Analysis of the behaviour of the long macromolecular chains, responsible for the viscoelastic effects: Microstructural analysis.

The theory of the continuous mediums is at the origin of the determinism principles. The approach of the continuous mediums does not distinguish the microstructure from material. The constitutive laws, formulated starting from the only principles quoted previously, are applicable to any type of fluid.

For the microstructural analysis, the step followed by these theories to obtain the constitutive law has for major aims:

- To clarify the different microstuctural mechanisms which constitute the formulation of the behaviour law;

- To provide a physical justification of the terms appearing in the behaviour law;

- To define the limits of the behaviour law. The modelling of the microstructure leads to various concepts according to whether the fluid is considered as a diluted polymer solution, concentrated polymer solution or even as dispersion:

- In the theory of diluted solutions, the long polymer chains are modelled by springs which characterize the deployment of the molecule. Hydrodynamic resistance due to the movement of the solvent (basic oil) and the Brownian resistance (thermal agitation), caused by the random movement of the molecule, are applied at each end of the spring. The polymer chains are supposed not to have any interaction between them, and are influenced only by the flow of solvent: Dumbbell theory [7],

- Theories of concentrated solution seek on the contrary to take into account the entanglements of the polymer chains. One regards the lubricant as a polymer network bathing in a solvent. During the flow, certain junctions disappear but evolution leads to the formulation of the constitutive equation: Network analysis.

The applicability of these various theories depends on the concentration and the length of the polymer chains. The model of Phan-Thien and Tanner belongs to the network analysis. This model was developed for the study of lubricant subjected to sudden requests. Under this type 


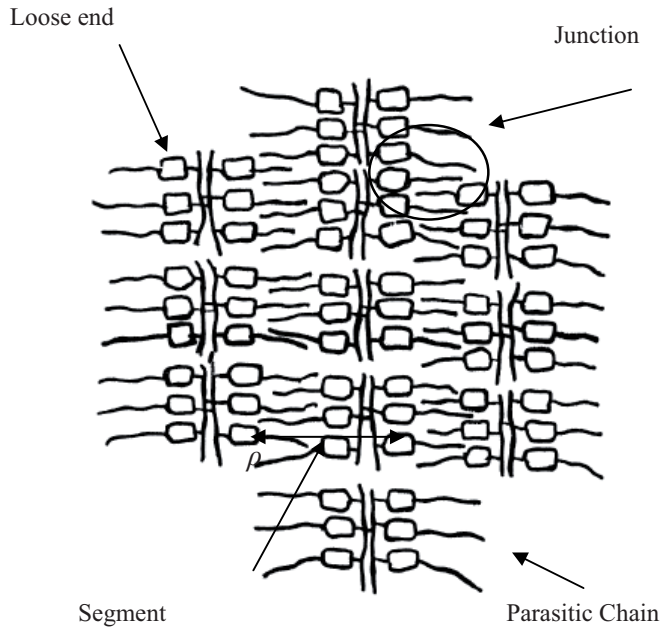

Fig. A.1. Representation of the network.

of stress, the lubricant loses its Newtonian character: its viscosity falls for raised rates of shearing, and stress overshoots are observed.

These properties thus show the possibility for interesting resemblances to the crude oil flow.

\section{A.1 Basic elements of the network analysis}

The network analysis of the macromolecular solids, the chemical articulation are described like points or junctions on the level of which, portions of molecules are always obliged to move together. By adapting this theory to the liquids, it is supposed that the junctions are not permanent but are continuously being created and destroyed. The principal approximation in the network analysis is the fact that the interactions between polymers are localized on the level of the junctions. A junction is a place of strong attraction between two isolated points which each one belongs to a different polymeric chain, and which are obliged to coincide.

A macromolecular chain joining two successive junctions is called "segment".

A macromolecular chain connected to only one junction is called "loose end".

A macromolecular chain not attached to the network is called "parasitic chain". The totality of the segments forms the molecular network. Let us recall that these junctions depend on time. Figure A.1 shows a part of the macromolecular network [8].

The modelling of this network is based on the following consideration: The polymer chains which are not segments, the parasitic chain and loose ends are omitted. So, Figure A.2 shows the model representation of the network.

There are six assumptions common to the network theories for both solids and liquids:

1) A concentrated polymer solution can be represented by a molecular network with polymer-polymer interactions occurring only as local interactions at isolated points

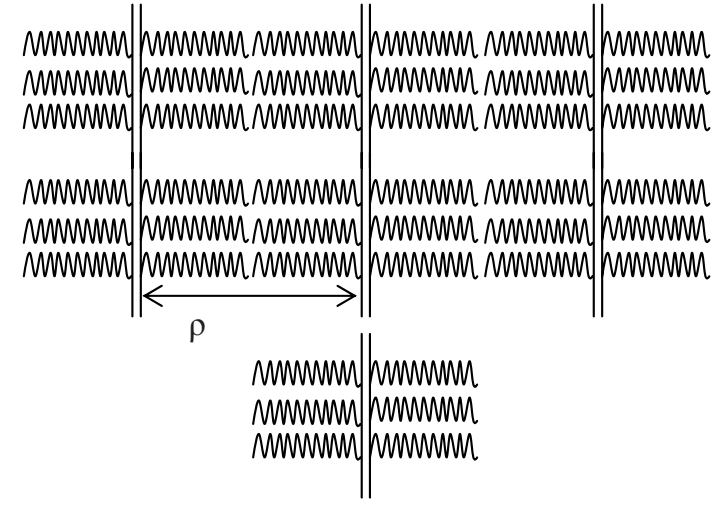

Fig. A.2. Modeling of the network.

along the chains. Only this network is responsible for the rheological behaviour of the material.

2) The polymer solution is assumed to be incompressible.

3) The network junctions move affinely.

4) The phase space distribution function for each segment is equilibrated at all times.

5) The polymer solution is subjected to either a homogeneous deformation or a homogeneous flow.

6) Each segment of the network can be modelled as a Gaussian chain.

These six assumptions cannot be modified without changing the theory basically. The network analysis for the solids rests essentially upon these six assumptions. For the liquids one adopts the following additional assumptions:

- Segments are lost and created during the flow. The network will consist of segments with a distribution of age.

- The stress is assumed to be the sum of contributions from all the existing segments at the present time, these having been created at various past times.

- It is supposed that the distribution function for the segments at the moment of creation is identical to the equilibrium distribution function for a freely jointed chain with no constraints on the end points.

- Segments can be characterized by only one positive integer $I$ that indicates the "complexity" of the segment.

For fluid not much is known about the mechanism for formation and loss of the temporary entanglements. This is why the theory focuses on the segments. It is expected that many different kinds of physical entanglements can be formed and that these will exhibit a wide range of degree of permanence. The idea of segment complexity is used to describe the relative permanence of different segments. One distinguishes the segments resulting from temporary physical entanglements with those formed between permanent chemical cross links. For the solids, the segments have all same complexity since they are all permanent. For the fluid the complexity of segment is tied to the topology of the "knot" formed by Brownian motion and which constitutes the junction. 


\section{A.2. Basic formulation}

Isothermal flow is considered. A chains part of molecule is located between two junctions. At each time, one can find chains of various lengths in an element of volume. The length of the chain is represented by the number density of subunits. $N_{i}$ is the number density of chains containing $i$ subunits ( $i$-chains). A chain is represented by its number of subunits and by the vector $\rho$ between its endpoints. $\Psi_{i}(\rho)$ is the distribution function of end-to-end vectors. $\Psi_{i}(\rho) \mathrm{d}^{3} \rho$ is the number of chains per unit volume having $i$ subunits and with a chain vector in a neighbourhood $\mathrm{d}^{3} \rho$ around $\rho[9]$.

The flow of the solution introduces a deformation of the network and then a destruction of junctions thus of chains, just like a formation of new junctions and chains. We are going to describe this process. For that, in a unit of volume, one has to take into account:

- The number of $i$-chains which leave and which enter the volume during the interval $\mathrm{d} t$,

- The rate of formation $k_{i}$ of these $i$-chains,

- The rate of disappearance $l_{i}$ of these $i$-chains,

- The total derivative of $\Psi$ is equal to the rate of creation minus the rate of destruction,

- The balance equation for the distribution function of the chains containing $i$ segments is:

$$
\frac{\mathrm{d} \psi_{i}}{\mathrm{~d} t}=\frac{\partial}{\partial \rho}\left(\psi_{i} \dot{\rho}\right)+k_{i}-l_{i}
$$

The model of Phan-Thien and Tanner is different from the other models of the network theory by taking for assumption a non-affine deformation of the polymer chains within solvent. The velocity $\dot{\rho}$ can be related to the endto-end vector $\rho$ through a transformation. If the motion of the junctions coincides with the motion of the material particles of the equivalent macroscopic continuum, then the transformation is linear: $\dot{\rho}=L \rho . L$ is the gradient speed tensor of the macroscopic flow $(\nabla \nu)$. The development is unchanged if the following non-affine deformation is employed:

$$
\dot{\rho}=(L-\zeta D) . \rho=\varsigma . \rho
$$

$D$ is the deformation rate tensor (the symmetrical part of $L) . \xi$ is a measure of the deformation-induced slip between the network and the continuum.

$\varsigma$ is the speed gradient tensor of the local flow.

It is necessary to specify the rates $k_{i}$ and $l_{i}$. The rate of disappearance is taken to be proportional to the number of the present chains:

$$
l_{i}=h_{i} \psi_{i}
$$

$h_{i}$ is a function of the chain size, configuration and local flow parameters. The formation of the segments follows a Brownian process. The rate of creation is proportional to the equilibrium distribution function $k_{i}=g_{i} \psi_{i, 0}$

It is assumed here that the flow will reduce the rate of creation of these junctions. This reduction is represented by a term that is first order in $\psi_{i}$ :

$$
k_{i}=g_{i} \psi_{i, 0}-\alpha_{i} \psi_{I}
$$

$g_{i}$ and $\alpha_{i}$ depend on the same variables as $h_{i}$.

At equilibrium, in the lack of any deformation, the rates of creation and destruction must be equal:

$$
h_{i, 0}=g_{i, 0}-\alpha_{i, 0}
$$

One then agrees to express $h_{i}$

$$
\begin{aligned}
h_{i} & =\left(g_{i}-\alpha_{i}\right)+p_{i} \\
p_{i, 0} & =0
\end{aligned}
$$

with the specific constitutive assumptions made above, the equilibrium equation for the distribution function (A.1) becomes:

$$
\frac{\mathrm{d} \psi_{i}}{\mathrm{~d} t}=\frac{\partial}{\partial \rho}\left(\psi_{i} * \varsigma . \rho\right)+g_{i}\left(\psi_{i, 0}-\psi_{i}\right)-p_{i} \psi_{i}
$$

The number density $N_{i}$ of $i$-chains is obtained by integration of the distribution function over the configuration space:

$$
N_{i}=\int \psi_{i} \mathrm{~d}^{3} \rho
$$

According to Phan-Thien and Tanner, it is assumed that the parameters $g_{i}, p i$ and $\xi$ depend at most on the distribution. In this case the integral of the equilibrium Equation (A.7) gives the kinetic equation:

$$
\frac{\mathrm{d} N_{i}}{\mathrm{~d} t}=g_{i}\left(N_{i, 0}-N_{i}\right)-p_{i} N_{i}
$$

It is convenient to define the fraction relating to the equilibrium of $i$-chains:

$$
x_{i}=\frac{N_{i}}{N_{i, 0}}
$$

One obtains then:

$$
\frac{\mathrm{d} x_{i}}{\mathrm{~d} t}=g_{i}\left(1-x_{i}\right)-p_{i} x_{i}
$$

The equation containing stresses is obtained by multiplying the equilibrium Equation (A.7) by the dyadic $\rho_{i} \rho_{i}$ and integrating over the configuration space:

$$
\frac{\mathrm{D}}{\mathrm{D} t}\left\langle\rho_{i} \rho_{i}\right\rangle=\frac{g_{i}}{x_{i}}\left(\left\langle\rho_{i, 0} \rho_{i, 0}\right\rangle-\left\langle\rho_{i} \rho_{i}\right\rangle\right)
$$

with $\frac{\mathrm{D}}{\mathrm{D} t}=\frac{\mathrm{d}}{\mathrm{d} t}()-\varsigma .()-() \cdot \varsigma^{t}=$ non affine differential operator.

The fluid being incompressible, the constraints are given with an arbitrary isotropic pressure $p_{i}$.

$$
\pi_{i}=\tau_{i}+p_{i}
$$

One wants to calculate $\tau_{i}$. The theory of the molecular kinetics provides the following expression:

$$
\tau_{i}=N_{i} \frac{3 k T}{i a^{3}}\left(\left\langle\rho_{i} \rho_{i}\right\rangle-\left\langle\rho_{i, 0} \rho_{i, 0}\right\rangle\right)
$$

where $a$ represents here the length of the chain. To determine $\left\langle\rho_{i, 0} \rho_{i, 0}\right\rangle$, it is necessary to define the distribution 
function of the segments at the initial moment taken as equilibrium. It is considered that the state of the initial constraints is isotropic. It is supposed that the function of distribution $\psi_{i, 0}$ has the following form:

$$
\begin{array}{r}
\psi_{i, 0}=\left(\frac{3}{2 \pi i a^{2}}\right)^{\frac{3}{2}} \exp \left(\frac{-3}{2 \pi i a^{2}} \rho^{2}\right) \\
\text { Hence }:\left\langle\rho_{i, 0} \rho_{i, 0}\right\rangle=\left(\frac{i a^{2}}{3}\right) I
\end{array}
$$

with $I$ the unit tensor and $\rho$ the end-to-end distance of a segment.

$k$ is the Boltzmann constant and $T$ the temperature.

By taking into account Equations (A.14) and (A.16), one can rewrite Equation (A.12):

$$
\frac{x_{i}}{g_{i}} \frac{\mathrm{D}}{\mathrm{D} t} \frac{\tau_{i}}{N_{i} k T}+\frac{\tau_{i}}{N_{i} k T}=\frac{x_{i}}{g_{i}} 2(1-\xi) D
$$

The total extra stress is given as the sum of the partial stresses:

$$
\tau=\sum \tau_{i}
$$

The relaxation time is given by:

$$
\lambda_{i}=\frac{1}{g_{i}+p_{i}}
$$

This relaxation time involves both the time constant of network destruction and the retardation of network build-up associated with the deformation.

\section{A.3 Phan-Thien and Tanner}

Two assumptions are made. The first assumption is a pseudo-steady state of the structure:

$$
\frac{\mathrm{d} N}{\mathrm{~d} t} \approx 0
$$

So, according to the Equation (9):

$$
N_{i} \approx \frac{g_{i}}{g_{i}+p_{i}} N_{i, 0}
$$

This assumes that structural transients are slow relative to a time scale of time equal to the relaxation time. One can rewrite the equation of stresses (A.7):

$$
\frac{\mathrm{D} \tau_{i}}{\mathrm{D} t}+\left(g_{i}+p_{i}\right) \tau_{i}=\frac{g_{i}}{g_{i}+p_{i}}\left(N_{i, 0} k T\right) 2(1-\xi) D
$$

The relaxation time can be written according to the stresses:

$$
\left(g_{i}+p_{i}\right)=\frac{\sigma_{i}\left(t r \tau_{i}\right)}{\lambda_{i, 0}}
$$

The second assumption, which is not fundamental in terms of basic behaviour, is that the functions $g_{i}$ and $g_{i}+$ $p_{i}$ are in a constant ratio:

$$
\frac{g_{i}}{g_{i}+p_{i}}=r_{i}=\text { const. }
$$

with Equations (23) and (24), Equation (22) can be written:

$$
\lambda_{i 0} \frac{\mathrm{D} \tau_{i}}{\mathrm{D} t}+\sigma_{i}\left(\operatorname{tr} \tau_{i}\right) \tau_{i}=2 \lambda_{i, 0} G_{i, 0} D
$$

with: $G_{i, 0}=r_{i}(1-\xi) N_{i, 0} k T$

One obtains the constitutive equation of the model of Phan-Thien and Tanner (PTT).

Phan-Thien uses: $\sigma_{i}=\exp \left(\in \frac{\operatorname{tr} \tau_{i}}{G_{i, 0}}\right)$

It is necessary to make a light modification to the model of Phan-Thien and Tanner in order to take into account the shear thinning effect. The shear thinning effect described the fall of viscosity at high rate of shearing. One obtains the constitutive equation of the model of modified Phan-Thien and Tanner (MPTT) [6]:

$$
\lambda_{i, 0} \frac{\mathrm{D} \tau_{i}}{\mathrm{D} t}+\sigma_{i}\left(\operatorname{tr} \tau_{i}\right) \tau_{i}=2 \eta_{\mathrm{m}} D
$$

with $\eta_{\mathrm{m}}$ the viscosity given by the law of Carreau [6]:

$$
\eta_{\mathrm{m}}=\eta_{\mathrm{p}} \frac{1+\xi(2-\xi) \lambda^{2} \dot{\gamma}^{2}}{\left(1+\Gamma^{2} \dot{\gamma}^{2}\right)^{(1-n) / 2}}
$$

where:

- $\eta_{\mathrm{p}}$ is the zero-rate shearing viscosity of the crude oil,

- $\lambda$ is the relaxation time of the fluid,

- $\Gamma$ is a time dimension parameter, fixed numerically to $11.08 \lambda$,

- $n$ is the power law index $(n<1)$.

\section{Appendix B: Velocity profile}

\section{B.1 Equation of motion}

In the case of a pipe of radius $R$ using $(r, \theta, z)$ as cylindrical coordinates and taking into account of the symmetry of the flow the Navier-Stokes equation writes:

$$
\frac{\mathrm{d} P}{\mathrm{~d} z}=\frac{1}{r} \frac{\mathrm{d}}{\mathrm{d} r}\left[\frac{r \eta \mathrm{d} V}{\mathrm{~d} r}\right]
$$

where $\eta$ represents the apparent viscosity. Thus:

$$
-a r=\frac{\mathrm{d}}{\mathrm{d} r}\left[r \eta \frac{\mathrm{d} V}{\mathrm{~d} r}\right] \text { with } a>0
$$

\section{B.2 Flow calculation for Krieger and Dougherty model}

Equation (B.2) can be written as :

$$
-a r=\frac{\mathrm{d}}{\mathrm{d} r}\left[r \tau_{r z}\right]
$$

as

$$
\eta=\eta_{\infty}+\frac{\eta_{0}-\eta_{\infty}}{1+\left[\frac{\tau}{\tau_{c}}\right]^{m}}
$$


We have:

$$
\tau=\left(\frac{\eta_{0}-\eta}{\eta-\eta_{\infty}}\right)^{\frac{1}{m}} \tau_{\mathrm{c}}
$$

After integration (B.3) becomes:

$$
-\frac{a r}{2}=\left(\frac{\eta_{0}-\eta}{\eta-\eta_{\infty}}\right)^{\frac{1}{m}} \tau_{\mathrm{c}}
$$

as $\tau_{\mathrm{c}}$ is negative we can write:

$$
\frac{a r}{2}=\left(\frac{\eta_{0}-\eta}{\eta-\eta_{\infty}}\right)^{\frac{1}{m}}\left|\tau_{\mathrm{c}}\right|
$$

\section{B.3 Velocity profile for $m$ variable}

For $m$ variable we obtain:

$$
\eta=\frac{\left(\tau_{\mathrm{c}}\right)^{m} \eta_{0}+\eta_{\infty}\left(\frac{a r}{2}\right)^{m}}{\left(\frac{a r}{2}\right)^{m}+\left(\tau_{\mathrm{c}}\right)^{m}}
$$

and

$$
\dot{\gamma}=\frac{|\tau|}{\eta}=\frac{-\frac{a r}{2}\left[\left(\frac{a r}{2}\right)^{m}+\left(\tau_{\mathrm{c}}\right)^{m}\right]}{\left[\left(\tau_{\mathrm{c}}\right)^{m} \eta_{0}+\eta_{\infty}\left(\frac{a r}{2}\right)^{m}\right]}
$$

Thus the velocity profile can be obtained from:

$$
\frac{\mathrm{d} V}{\mathrm{~d} r}=\frac{-\frac{a r}{2}\left[\left(\frac{a r}{2}\right)^{m}+\left(\tau_{\mathrm{c}}\right)^{m}\right]}{\left[\left(\tau_{\mathrm{c}}\right)^{m} \eta_{0}+\eta_{\infty}\left(\frac{a r}{2}\right)^{m}\right]}
$$

For $m=1$ we have:

$$
\frac{\mathrm{d} V}{\mathrm{~d} r}=\frac{-\frac{a r}{2}\left[\left(\frac{a r}{2}\right)+\left(\tau_{\mathrm{c}}\right)\right]}{\left[\left(\tau_{\mathrm{c}}\right)_{0}^{\eta}+\eta_{\infty}\left(\frac{a r}{2}\right)\right]}
$$

For $m=1$ the velocity profile is obtained after integrating (B.11). Setting:

$$
A=\frac{2 \eta_{0}\left|\tau_{\mathrm{c}}\right|}{a \eta_{\infty}} \text { and } A A=\frac{-\frac{a r}{2}\left[\left(\frac{a r}{2}\right)^{m}+\left(\tau_{\mathrm{c}}\right)^{m}\right]}{\left[\left(\tau_{\mathrm{c}}\right)^{m} \eta_{0}+\eta_{\infty}\left(\frac{a r}{2}\right)^{m}\right]}
$$

we obtain:

$$
\begin{array}{r}
V(r)=\frac{a}{4 \eta_{\infty}}\left(R^{2}-r^{2}\right)+\left[\frac{a A}{2 \eta_{\infty}}-\frac{\left|\tau_{\mathrm{c}}\right|}{\eta_{\infty}}\right](r-R) \\
+\left[\frac{\left|\tau_{\mathrm{c}}\right| A}{\eta_{\infty}}-\frac{a A^{2}}{2 \eta_{\infty}}\right] \ln \left|\left[\frac{r+A}{A+R}\right]\right|
\end{array}
$$

\section{B.4 Modified Krieger and Dougherty model}

From (B.2) and

$$
\eta=\left[\eta_{\infty}+\frac{\eta_{0}-\eta_{\infty}}{1+\left[\frac{\tau}{\tau_{c}}\left(\frac{1}{a^{\frac{2}{3}}}\right)\right]^{m}}\right] a^{\frac{2}{3}}
$$

We have:

$$
\tau=\left(\frac{\eta_{0}-\left(\eta / a^{\frac{2}{3}}\right)}{\left(\eta / a^{\frac{2}{3}}\right)-\eta_{\infty}}\right)^{\frac{1}{m}} \tau_{\mathrm{c}}\left(a^{\frac{2}{3}}\right)
$$

After integration:

$$
-\frac{a r}{2}=\left(\frac{\eta_{0}-\left(\eta / a^{\frac{2}{3}}\right)}{\left(\eta / a^{\frac{2}{3}}\right)-\eta_{\infty}}\right)^{\frac{1}{m}} \tau_{\mathrm{c}}\left(a^{\frac{2}{3}}\right)
$$

As $\tau_{\mathrm{c}}$ is negative we can write:

$$
\frac{a r}{2}=\left(\frac{\eta_{0}-\left(\eta / a^{\frac{2}{3}}\right)}{\left(\eta / a^{\frac{2}{3}}\right)-\eta_{\infty}}\right)^{\frac{1}{m}}\left|\tau_{\mathrm{c}}\right|\left(a^{\frac{2}{3}}\right)
$$

\section{B.5 Velocity profile $\mathrm{m}$ variable modified Krieger and Dougherty model}

For $m$ variable we can obtain:

$$
\eta=\left[\frac{\left(\tau_{\mathrm{c}}\right) \eta_{0}+\eta_{\infty}\left(\frac{a^{\frac{1}{3}} r}{2}\right)}{\left(\frac{a^{\frac{1}{3}} r}{2}\right)+\left(\tau_{\mathrm{c}}\right)}\right]\left(a^{\frac{2}{3}}\right)
$$

and:

$$
\dot{\gamma}=\frac{|\tau|}{\eta}=\frac{-\frac{a^{\frac{1}{3}} r}{2}\left[\left(\frac{a^{\frac{1}{3}} r}{2}\right)+\left(\tau_{\mathrm{c}}\right)\right]}{\left[\left(\tau_{\mathrm{c}}\right) \eta_{0}+\eta_{\infty}\left(\frac{a^{\frac{1}{3}} r}{2}\right)\right]}
$$

Thus the velocity profile writes:

$$
\frac{\mathrm{d} V}{\mathrm{~d} r}=\frac{-\frac{a^{\frac{1}{3}} r}{2}\left[\left(\frac{a^{\frac{1}{3}} r}{2}\right)+\left(\tau_{\mathrm{c}}\right)\right]}{\left[\left(\tau_{\mathrm{c}}\right) \eta_{0}+\eta_{\infty}\left(\frac{a^{\frac{1}{3}} r}{2}\right)\right]}
$$

Setting :

$$
A=\frac{2 \eta_{0}\left|\tau_{\mathrm{c}}\right|}{a^{\frac{1}{3}} \eta_{\infty}}
$$

$$
\begin{array}{r}
V(r)=\frac{a^{\frac{1}{3}}}{4 \eta_{\infty}}\left(R^{2}-r^{2}\right)+\left[\frac{a^{\frac{1}{3}} A}{2 \eta_{\infty}}-\frac{\left|\tau_{\mathrm{c}}\right|}{\eta_{\infty}}\right](r-R) \\
+\left[\frac{\left|\tau_{\mathrm{c}}\right| A}{\eta_{\infty}}-\frac{a^{\frac{1}{3}} A^{2}}{2 \eta_{\infty}}\right] \ln \left|\left[\frac{r+A}{A+R}\right]\right|
\end{array}
$$




\section{References}

[1] S. Tebbal, R.D. Kané, Review of critical factors affecting crude corrosivity, Corrosion 96, 607, The NACE International Annual Conference and Exposition, Texas, USA, 1996

[2] A. Sinquin, T. Palermo, Y. Peysson, Rheological and flow properties of gas hydrate suspensions, Oil \& Gas Science and Technology, Rev. IFP 59 (2004) 41-57

[3] M. Kane, M. Djabourov, Rheology and structure of waxy crude oils in quiescent and under shearing conditions, J. Fuel (2004) 1591-1605

[4] M. Kane, M. Djabourov, Rheology and structure of waxy crude oils in quiescent and under shearing conditions, J. Fuel 82 (2004) 1591-1605

[5] A. Makria, Comportement rhéologique des émulsions concentrées de pétrole brut lourd dans l'eau, École nationale supérieure de techniques avancées, Rapport de recherche, 194, novembre 1984

[6] P. Ehret, Contribution à l'étude du comportement de mécanismes lubrifiés sous chargement transitoire, Mémoire de doctorat, Institut National des Sciences Appliquées de Lyon, 1993

[7] N. Phan Thien, R.I. Tanner, A new constitutive equation derived from network theory, J. Non-Newtonian Fluid Mech. 2 (1977) 353-365

[8] B. Bou-Saï, A new modelling of human joint lubrication subject to shock loading, Revue Européenne des Éléments-Finis, Numéro Spécial Biomécanique des Chocs, 14 (2005) 397-420

[9] A. Amblard, B. Bou-Said, Modelling of the blood flow in an aorta: an attempt, The MPTT and Modified MPTT models, J. Eng. Tribology IME 48 (2005) 381-387 